\title{
VULNERABILIDAD SOCIOECONÓMICA, CAMBIO CLIMÁTICO E INCREMENTO DEL NIVEL DEL MAR EN TUXPAN, VERACRUZ
}

\section{SOCIOECONOMIC VULNERABILITY, CLIMATE CHANGE AND INCREASE IN THE LEVEL OF THE SEA IN TUXPAN, VERACRUZ}

\author{
Rodrigo Tovar Cabañas \\ Universidad Autónoma de Tlaxcala, México. \\ rod_geo77@hotmail.com | 0000-0003-4873-2502
}

\begin{abstract}
Para citar este artículo: Tovar Cabañas, R. (2021). Vulnerabilidad socioeconómica, cambio climático e incremento del nivel del mar en Tuxpan, Veracruz. Entorno Geográfico, (22), 79-93. https://doi.org/10.25100/eg.v0i22.11399
\end{abstract}

\section{Resumen}

La vulnerabilidad socioeconómica es un tema prioritario debido a los efectos del cambio climático, el aumento de entropía de los distintos factores climáticos, ligado al aumento de desastres repercute en el desarrollo sustentable de los municipios costeros. El objetivo consistió en mostrar los impactos socioeconómicos que puede sufrir el municipio de Tuxpan, Veracruz, bajo el escenario de un incremento súbito en el nivel del mar de cinco metros. La matriz metodológica consistió en espacializar y cualificar a los grupos vulnerables. Resultados: sí el municipio de Tuxpan sufriera una inundación de cinco metros sobre el nivel del mar, $20 \%$ de su territorio quedaría anegado.

Palabras Clave: Vulnerabilidad Socioeconómica, Impacto Económico, Índice de Vulnerabilidad Social, Geografía Litoral, Cambio Climático y Nivel del Mar.

\begin{abstract}
The objective of the research is to expose the socioeconomic impacts in Tuxpan, Veracruz, considering a sudden increase in sea level of five meters. The multidisciplinary methodology consisted of locating, territorially quantifying the vulnerable groups that are currently within the danger zone and the second consisted of qualifying, through fieldwork, the specificities of the same. the most pressing results were: the municipality of Tuxpan suffered a flood of five meters above sea level, $20 \%$ of its territory would be flooded, that is, $187 \mathrm{~km}^{2}$ of its demarcation present a certain degree of socioeconomic vulnerability to sea level rise.

Keywords: Socioeconomic Vulnerability, Economic Impact, Social Vulnerability Index, Coastal Geography, Climate Change and Sea Level.
\end{abstract}




\section{Introducción}

La Secretaría de Protección Civil (SPC, 2011), desde el enfoque geotécnico del desastre, hace diez años realizó diferentes análisis gravitacionales de riesgos para el municipio de Tuxpan. En general, ese tipo de atlas de riesgos presentan duplicidad de información, sobre todo en torno a los datos de vivienda. La documentación de gabinete suele ser escasa, por ejemplo, en dicho atlas (SPC, 2011) no se consultaron los registros del proyecto Desinventar, el cual contiene registros de desastres desde 1970. De modo que este tipo de atlas dan la impresión de ser más una enciclopedia, puesto que, en el lugar donde debería existir información puntual, sobre los fenómenos acaecidos en el lugar bajo estudio, se apostan anécdotas fuera de contexto. El citado Atlas de Riesgos de Tuxpan es en realidad una mezcla de una monografía geográfica con un atlas geológico, donde la falta de trabajo interdisciplinario termina en recomendaciones muy generales.

Se advierten errores epistemológicos y metodológicos de consideración, por ejemplo, en cuanto a los riesgos antropogénicos de la ciudad de Tuxpan, epistemológicamente, el Atlas omite buena cantidad de estos, pero omite la delincuencia organizada, la violencia, el narcotráfico, los reclusorios, las jaurías de perros y las cantinas. Ese error teórico se debe a que sus hacedores imitaron lo realizado en los atlas de riesgos europeos, como el de Veyret et al. (2013), es decir, al no tropicalizar o intronizar los contenidos asumen que los taludes inestables de las carretas de Suiza, presentan el mismo grado de riesgo que los de Tuxpan, lo cual epistemológicamente llevó a considerar más a un talud carretero que a un foco rojo como peligro socioorganizativo en Tuxpan. Esto no sólo se cometió en torno a los peligros socioorganizativos sino también en el caso de los peligros geológicos, puesto que en el afán de estandarizar lugares geográficamente diferentes, sólo se consideraron los riesgos por: sismos, vulcanismos, procesos de remoción en masa, hundimientos y erosión en Tuxpan, omitiendo el riesgo por radiación de cobalto inducido por el mal manejo que se tuvo de este material durante la década de los años 1980, o por una posible explosión de la central nucleoeléctrica de Laguna Verde, así como el riesgo por tremores derivados del fracking o fractura hidráulica, la cual podría desecar las zonas lacustres ubicadas al este de la demarcación municipal (zona de karst subterráneo).

Respecto a los peligros sanitarios no contemplados en el Atlas de Riesgos de Tuxpan son los que tienen que ver con las enfermedades oculares, tales como la silicosis y la conjuntivitis (Galván, 2013). Por otra parte, el modelo de erosión propuesto en el Atlas antes dicho no fue tropicalizado, es decir, los factores de erosión podrían asumirse como correctos siempre y cuando se apliquen a zonas de denudación y no en zonas de acumulación (caso de Tuxpan). Tuxpan se ubica en zona costera a nivel del mar, por ende, es un lugar de acumulación, el riesgo no está en la erosión sino en el asolvamiento del río Tuxpan, el cual en algunos sitios llega a tener más de 15 metros de espesor (Vorderwinkler, 1983).

Otra inconsistencia del Atlas de Riesgos de Tuxpan es de orden metodológico, debido a que los observadores del fenómeno bajo estudio proceden en su mayoría del área de ciencias de la Tierra, estos trasladaron la escala regional, de observación de los procesos geológicos, al campo de estudio de los desastres. Este hecho, sin precedentes en la historia de la filosofía de las ciencias, es la razón 
de que, pese a que la resolución de los materiales cartográficos metodológicamente se procesó en metros (escala urbana), los mapas de riesgo se construyeron a escalas regionales de 1: 250,000, es decir en kilómetros. Lo cual es un error de precisión serio.

Con frecuencia los estudios sobre desastres se realizan de forma unilateral, es decir, unos trabajos sólo abordan las amenazas (como el caso del Atlas de Riesgos de Tuxpan) y otros sólo las vulnerabilidades. Por lo que comúnmente, quienes abordan sólo la parte de las amenazas suelen cometer errores epistemológicos al asumir, como existente, únicamente a la vulnerabilidad económica, omitiendo más de 15 variables sociodemográficas involucradas con los procesos de vulnerabilidad, de modo que se confunde la vulnerabilidad económica con el índice de vulnerabilidad social.

Para subsanar estas lagunas es menester contextualizar la cronología de los principales desastres acaecidos en el territorio del municipio de Tuxpan para posteriormente describir su ubicación geográfica y la relación que guarda con los fenómenos hidrometeorológicos extremos bajo la coyuntura de cambio climático y su índice de vulnerabilidad social más reciente, de modo que se puedan valorar en su justa dimensión los impactos económicos y sociales en caso de que el nivel del mar, durante las próximas décadas, llegará a subir ocasionalmente hasta la cota de cinco msnm. Los asentamientos en Tuxpan, desde sus orígenes prehispánicos, han padecido sequías e inundaciones, sin embargo, se desconocen los avatares del pueblo huasteco en dicho lugar. En 1610 se funda la pesquería de Tabuco, como se le conocía a Tuxpan en ese tiempo, pero es hasta el siglo XIX que se tienen las primeras noticias en torno a desastres de corte hidrometeorológico, por ejemplo: en 1878 la sequía fue tan fuerte que los habitantes de Tuxpan tuvieron que importar maíz de Estados Unidos, el mismo fenómeno natural ocurrió en 1896, en tanto que en 1899 las heladas destruyeron las siembras de maíz, frijol, pastos para ganado, platanares, caña de azúcar y vainilla, solo sobrevivió el cultivo de tabaco, pues de este cultivo sólo se perdieron 60 hectáreas; otros episodios corresponden a desastres agricolas: como la plaga de langosta que ocurrió en 1885, (Escobar, 2004).

Llama poderosamente la atención que el primer acercamiento científico y sistemático al estudio integral de las inundaciones de Tuxpan se realizara hasta el siglo XXI. Alarcón (2011), fue quien caracterizó geográficamente las áreas susceptibles a inundarse en la cuenca baja del río Tuxpan, de la que comenta que la margen derecha del río Tuxpan particularmente es la que más se anega, por lo que considera a dicha margen como la zona de mayor riesgo de dicho municipio, puesto que al proyectar escenarios de riesgo por inundación hasta el nivel de los 2 metros de altura, la configuración de la zona vulnerable forma un amplio corredor sinuoso que cubre una zona de inundación de aproximadamente $266 \mathrm{~km}^{2}$. Advierte que ese nivel de inundación, llega a penetrar hasta $51.5 \mathrm{~km}$ tierra adentro sobre las tierras bajas. Al respecto, Vergara, et. al. (2011), señalan que tales zonas de inundación afectan a 84 localidades de los municipios de Tuxpan y Temapache. Sobre la población refieren que el $6 \%$ presenta marginación alta, 12\% de marginación media y $82 \%$ marginación baja, y que el $40 \%$ de la población afectada está dispuesta a reubicarse.

Un año despues Ellis et. al. (2012), mediante un estudio de modelado de cuencas hidrológicas, demuestran que la superficie de inundación varía entre $522 \mathrm{~km}^{2}$, para periodos de retorno de dos años, y $554 \mathrm{~km}^{2}$, para periodos de retorno de 100 años. Donde las principales afectaciones 
impactan directamente a $343 \mathrm{~km}^{2}$ de tierras agrícolas y alrededor de $15 \mathrm{~km}^{2}$ de áreas urbanas, de estas ultimas $6.3 \mathrm{~km}^{2}$ corresponden a las áreas urbanas, de la demarcación municipal de Tuxpan, que cada dos años sufren inundaciones, las cuales podrían crecer hasta $8.4 \mathrm{~km}^{2}$ (33\% más de lo habitual), si se considera un periodo de retorno de 100 años.

Por otra parte, en el último medio siglo, de acuerdo con los registros de Desinventar (LARED, 2019), la ciudad y puerto de Tuxpan, Veracruz de 1970 al año 2013 ha experimentado 105 desastres de consideración estatal y nacional. Como se puede observar en la (Tabla 1) el 80.9\% de ellos corresponden a eventos de corte meteorológico, en orden de prelación se pueden describir como sigue: $35 \%$ vendavales, $19 \%$ inundaciones, $16 \%$ aguaceros o tempestades de consideración y $9 \%$ marejadas, los cuales, en conjunto, en términos históricos, dejaron más de 425,000 personas afectadas, de los cuales alrededor de 500 tuvieron que ser evacuadas en su momento.

Tabla 1. Tuxpan: ocurrencia de desastres de 1970 a 2013 en porcentaje.

\begin{tabular}{|l|c|l|c|}
\hline \multicolumn{1}{|c|}{ Tipo de evento } & \% & Tipo de evento & \% \\
\hline Vendaval & 35 & Sequía & 3 \\
\hline Inundación & 19 & Incendio & 2 \\
\hline Marejada & 9 & Explosión & 2 \\
\hline Lluvias & 9 & Contaminación & 2 \\
\hline Tempestad & 7 & Epidemia & 2 \\
\hline Helada & 1 & Escape & 2 \\
\hline Avenida torrencial & 1 & Biológico & 1 \\
\hline Onda fría & 1 & Accidente & 1 \\
\hline Deslizamiento & 5 & & \\
\hline
\end{tabular}

Fuente: Elaboración propia con base en Desinventar, 2019.

Para el futuro cercano de Tuxpan, es útil saber que el promedio de retorno de los fenómenos hidrometeorológicos más intensos, durante el siglo XX oscila entre el 1.3 y los 2 años (LARED, 2019), lo que significa que en el último medio siglo, el mayor lapso de tiempo que Tuxpan ha estado libre de algún fenómeno hidrometeorológico es de dos años, por lo que es muy importante dar a conocer a detalle las zonas que presenten el mayor índice de vulnerabilidad social y que además sean propensas a riesgo por concepto de inundación. Como se puede apreciar, el pricincipal peligro documentado que aqueja a la ciudad de Tuxpan está asociado a los fenómenos hidrometeorológicos.

Dichos antecedentes han contribuido a entender cómo el hundimiento costero que experimenta el centro de Tuxpan, más el azolvamiento asociado a los sedimentos y la pérdida de la cubierta forestal aguas arriba, agravan el riesgo de sufrir inundaciones de respuesta rápida de hasta 1.5 metros de tirante, como ocurrió en 2013, a consecuencia de la fuerte temporada de lluvias de ese año, sobre todo en las colonias periféricas y rancherías. Sin embargo, existen otros peligros geológicos (campos de fractura hidráulica) que no han sido visibilizados, por ejemplo la perforación de un acuifero confinado, podría provocar una crecida de carácter torrencial. Más allá de las recurrentes inundaciones anuales en la cuenca del río Tuxpan, es el silencio de más de dos 
décadas en el periodo de retorno del último huracán, categoría cuatro o superior, lo que debe llamar a la alerta.

Pese a lo anterior, la dislocación cartográfica con la que se han elaborado los mapas de riesgo de Tuxpan, aunado al aumento de infraestructura social (no adaptada a las inundaciones), puede hacer pensar, que Tuxpan es un lugar de riesgo moderado. Esta subestimación conduce a una incomprensión de los factores físicos y sociales, de modo que sus coropletas, si bien es cierto que pueden ser empleadas para la predicción de escenarios de riesgo por fenómenos hidrometeorológicos, de gran valor para la concientización de sus habitantes sobre la peligrosidad de determinados fenómenos naturales, les hace falta una mejor yuxtaposición tendiente a resolver los cómo de la planificación y el desarrollo sustentable que exige la actual coyuntura en materia de cambio climático.

Pese a que año tras año Tuxpan sufre inundaciones de consideración, las cuales dejan notar cómo la anegación de las calles es la variable independiente, el Puerto de Tuxpan sólo cuenta con el Atlas de Riesgos de Tuxpan (SPC, 2011). Por lo tanto, aún siguen sin resolverse ciertas preguntas vinculadas con la protección civil, tal como: ¿Cuánto ha crecido la mancha urbana en peligro de inundación de Tuxpan en caso de que el nivel del mar subiera súbitamente cinco metros?, ¿Qué características socioeconómicas presenta la población que está en dicha zona de riesgo ante un escenario como el descrito? Por lo tanto, el presente estudio busca dar respuesta a estas interrogantes que son pertinentes dada la complejidad actual de un sistema que está en constante crecimiento y cuyos riesgos se potencializan de no contar con herramientas sólidas de planeación y análisis territorial para la toma de decisiones tendientes a la prevención y adaptación de las zonas más sensibles a las alteraciones pluviométricas y potamológicas vinculadas con la era del Antropoceno y el cambio climático.

\section{Incremento del nivel del Mar}

Cuantificar el promedio del nivel mar es complejo puesto que las distintas herramientas registran variaciones de consideración debido a que algunas áreas costeras se levantan otras se deprimen, por lo que los cambios del nivel del mar todavía no son incuestionables. Empero, en investigaciones recientes se ha observado que del Dryas Reciente, el mar se elevó cuatro metros por siglo Webster et al. (2004), a escala histórica, según el Grupo Intergubernamental de Expertos sobre el Cambio Climático (IPCC, por sus siglas en inglés), dicha tasa es de $0.5 \mathrm{~mm} / \mathrm{año}$ para el final del epipaleolítico y de $0.1 \mathrm{~mm} /$ año para los últimos tres mil años (Kopp et al., 2016). Recientemente en Holanda, el ascenso se ha estimado en $1.5 \mathrm{~mm} / \mathrm{año}$. Por lo que, al finalizar este siglo se espera que el aumento del nivel del mar no sea simétrico en las diferentes latitudes del planeta, por lo que su impacto será muy variable en las distintas regiones costeras del mundo. El IPCC (2007), en términos científicos considera un ascenso del nivel del mar dentro del rango de $\operatorname{los} 19.0$ a $57.9 \mathrm{~cm}$ hacia 2100; otros autores amplían dicho rango a 1.3 metros para el mismo periodo (Grinsted et al., 2010). Aunque ciertamente, es imposible descartar las variaciones regionales provocadas por fenómenos hidrometeorológicos extremos, entre otros de orden geoingenieril. Otros investigadores han escenificado costas anegadas hasta las cotas actuales de cinco y 10 msnm, como Cruz (2016), quien a partir de un índice de vulnerabilidad costera ubica 
al municipio de Tuxpan, Veracruz, dentro del grupo de los moderadamente vulnerables de todo el estado, dado que cuenta con seis localidades con un índice de vulnerabilidad costera por encima del promedio, estas son por orden de prelación: Villa Mar Chile Frío, Barra Norte, San Antonio, Playa Emiliano Zapata, Tampamachoco y Linda Vista. Bajo estas consideraciones mareológicas, potamológicas, geológicas e ingenieriles se presenta un escenario para la cota cinco metros de anegación del municipio de Tuxpan.

\section{Área de estudio}

Geográficamente el municipio de Tuxpan se asienta sobre la Llanura Costera del Golfo de México, al noreste de la Sierra Norte de Puebla, en términos geológicos su suelo se constituye de depósitos de llanuras aluviales, principalmente sobre el curso inferior de Tuxpan, estos depositos frecuentemente están divididos por áreas con predominio de limo y por áreas con predominio de arena; en otros lugares existe aluvión indivisible, excepto sobre las áreas planas de marea, en donde hay elementos heterogéneos de arcilla, limo, arena, grava y materia orgánica (Carreón, 1990), además de limo y arena de origen calcáreo de color gris oscuro a marrón oscuro, la grava a lo largo del río Tuxpan incluye rocas sedimentarias del periodo cretácico.

En cuanto al clima, de acuerdo a la elevación topográfica, la cuenca del río Tuxpan presenta siete variantes: en la costa el clima es cálido subhúmedo; en la zona central es de tipo monzónico, en ambos casos la precipitación anual es menor de $700 \mathrm{~mm}$; en las sierras predominan los climas semicálidos húmedos (con precipitación anual mayor a $1500 \mathrm{~mm}$ ); templados húmedos y semifríos húmedos, ambos con precipitaciones menores a los $700 \mathrm{~mm}$ ). En sintesis, para el caso de las inundaciones, es útil señalar que la precipitación media de toda la cuenca es de $1458.5 \mathrm{~mm}$ (DOF, 2018). Dicha localidad pertenece a uno de los 212 municipios del estado de Veracruz, el cual se ubica al norte, en la región Huasteca Alta a una altitud promedio de $10 \mathrm{msnm}$ (Figura 1), con una extensión territorial de 1,062 km² y una población al censo de INEGI (2020) de 154,600 habitantes.

\section{Tuxpan a cinco metros bajo las aguas del Mar}

A penas hace una década que en México el gobierno federal impulsó una iniciativa para abordar el tema del cambio climático, por lo que es de comprender que los escenarios y estimaciones a nivel local aún son escasos, por ejemplo, en 2010, el Instituto de Ciencias del Mar y Limnología de la UNAM y las Universidades de Campeche y Autónoma Metropolitana, hicieron el esfuerzo para indicar las tendencias y comportamiento del nivel del mar, gracias a ello se sabe que frente a Tuxpan el nivel del mar, en promedio, subió su nivel $9 \mathrm{~cm}$ de 1961 a 1988, y aunque posteriormente esa tasa se ha incrementado, (Zavala et al., 2011) estiman que en dicho lugar el nivel asciende $3 \mathrm{~cm}$ por década. Esa investigación enriquece parte de estas indagaciones, ligadas al desarrollo local sustentable.

Luego de emplear un método de fotogrametría satelital para obtener las curvas de nivel ${ }^{1}$ que sirvieron para ubicar y delimitar las zonas del territorio del municipio de Tuxpan que quedarían bajo el agua del mar, en caso de que éste subiera cinco m su nivel en las próximas décadas, se

\footnotetext{
${ }^{1}$ Con una equidistancia de $20 \mathrm{~m}$ se construyó, una malla de un $\mathrm{km}^{2}$, en formato shape, la cual se exportó como curva a formato KML para su tratamiento en Google Earth y en 3D-Route Builder y así tabular las coordenadas "x, y, z". Con ayuda de TCX-Converter la tabla se exportó a formato CSV para poder generar una interpolación desde Quikgrid. Las isolíneas resultantes se exportaron a DXF para su tratamiento cartográfico en GvSIG. Todo el proceso se iteró más de 20 veces.
} 
llegó a los resultados siguientes: de los mil $\mathrm{km}^{2}$ con los que cuenta el municipio de Tuxpan (INEGI, 2009) $187.33 \mathrm{~km}^{2}$ quedan por debajo de la cota de cinco msnm, lo que representa el $19.65 \%$ del total municipal. Tomando en cuenta únicamente el área urbana total del municipio (aproximadamente 2000 hectáreas), 18.9\% de su infraestructura urbana queda por debajo de la cota de cinco msnm.

Fuente: Elaboración propia con base en (INEGI, 2009).

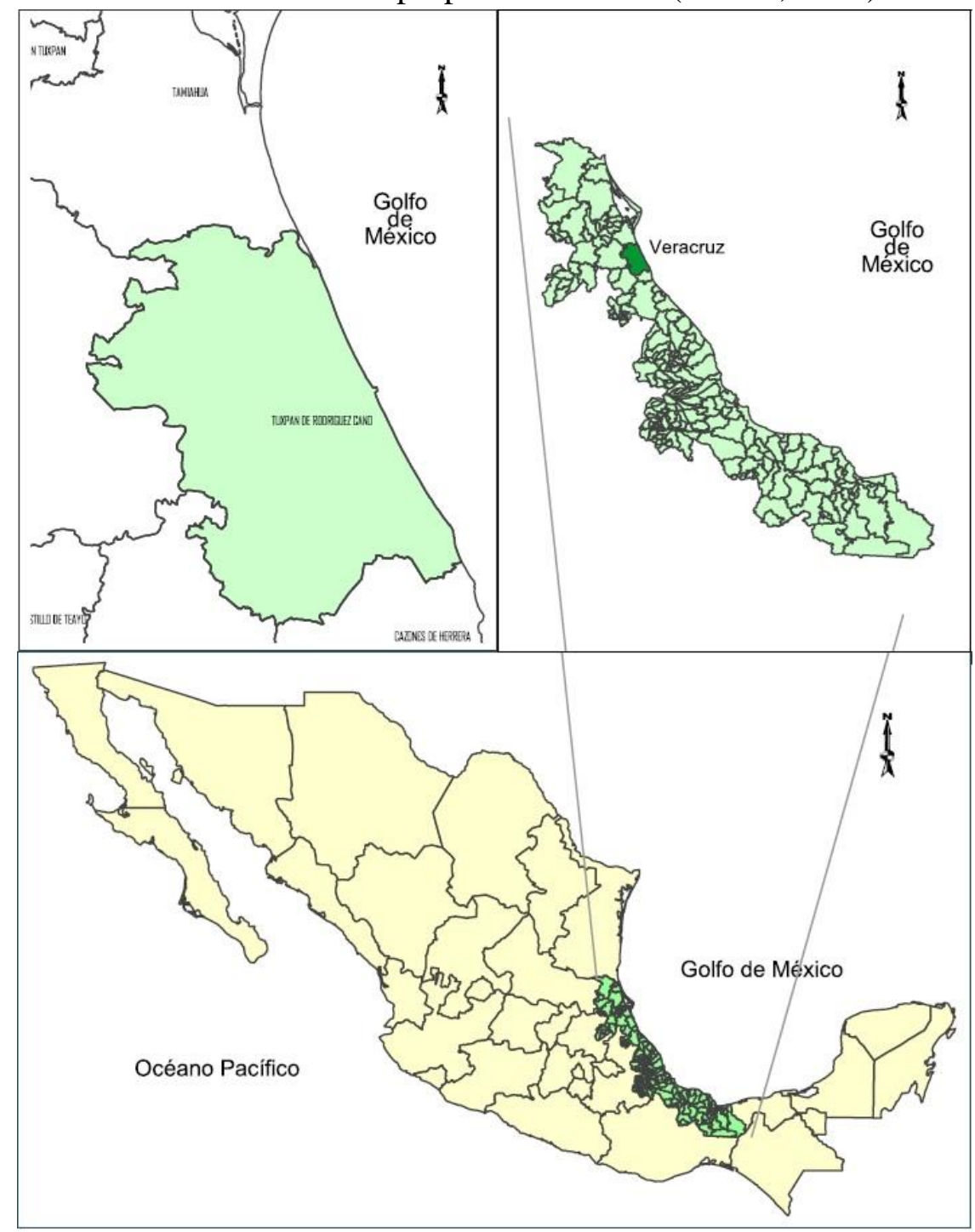

Figura 1. Ubicación del municipio de Tuxpan.

Prácticamente la quinta parte de esas dos mil hectáreas urbanizadas ${ }^{2}$, desde ya están en riesgo de inundación en la ciudad de Tuxpan (Figura 2), se localizan al oriente de la ciudad, al sureste del Aeropuerto Nacional Fausto Vega Santander, sobre las cuales se investigaron los principales impactos socioculturales y económicos que se podrían derivar a raíz de una subida del nivel del

\footnotetext{
${ }^{2}$ Cognitivamente eso equivale cuatro mil canchas de balompié o futbol.
} 
mar de cinco metros durante algún fenómeno hidrometeorológico, producido por las alteraciones pluviométricas que está generando el cambio climático.

Fuente: Cálculos de los autores con base en datos proporcionados por Google Earth 2019 -SIO, NOAA, US Navy, NGA y GEBCO.

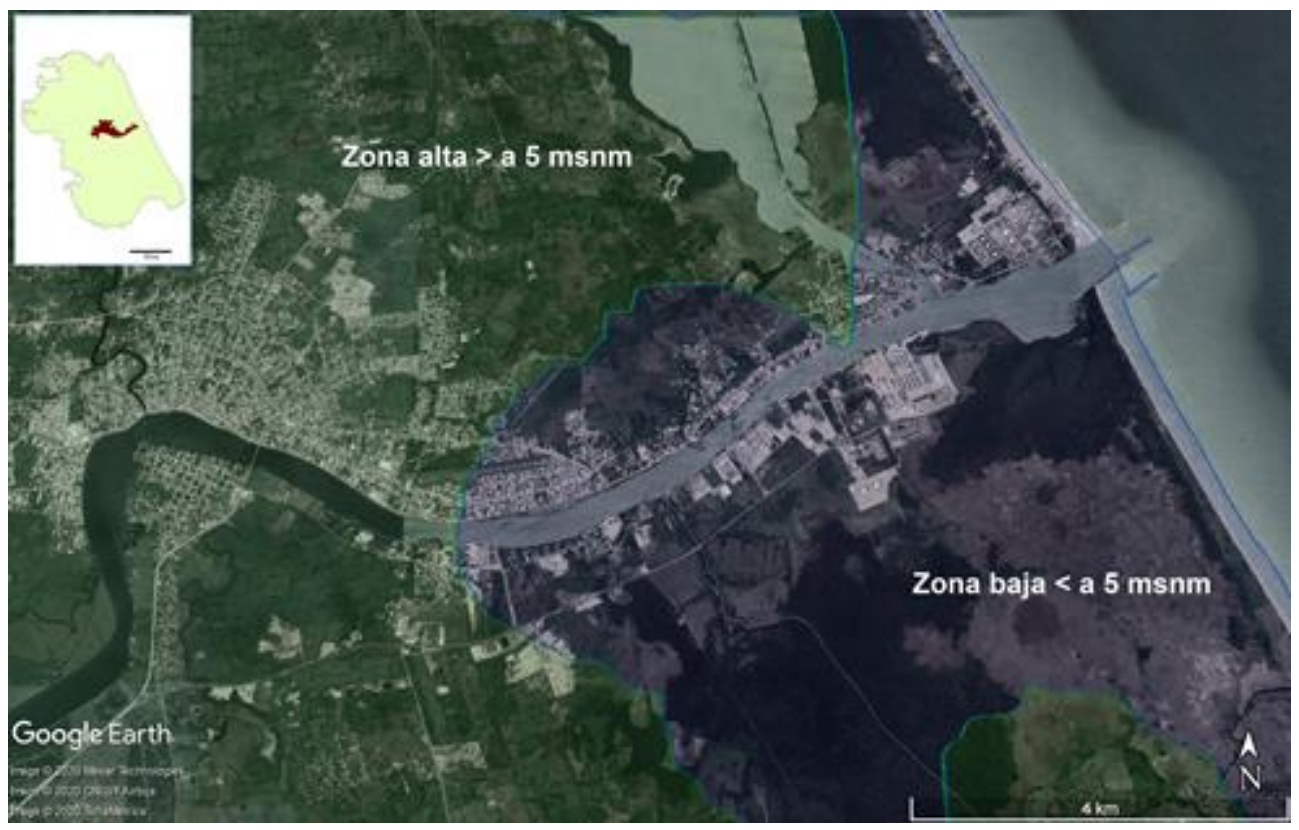

Figura 2. Principales zonas bajas del municipio de Tuxpan.

\section{Impactos económicos ante el escenario de incremento del Mar}

Una de las principales afectaciones del cambio climático en las zonas costeras del Golfo de México está relacionada con el incremento del nivel del mar, la cual, económicamente, se puede estimar ${ }^{3}$ a partir de la cuantificación del Producto Interno Bruto (PIB) municipal y por la estimación del valor de los servicios ambientales que aportan ciertos ecosistemas. En ese sentido, en este apartado, se reflexiona en torno a las cifras económicas del municipio de Tuxpan.

Conforme a González y Gallegos (2014), la distribución porcentual del Producto Interno Bruto, del año 2010, de los municipios de Veracruz, por región es como sigue: Huasteca Alta 5\%; Huasteca Baja 5\%; Las Montañas 16\%; Nautla 4\%; Olmeca 17\%; Papaloapan 6\%; Región Capital 16\%; Región de los Tuxtlas 3\%. Sotavento 19\%; y Totonaca 9\% (Tabla 2). Si consideramos el PIB estatal ${ }^{4}$ que arroja INEGI (2012), la región de la Huasteca Alta, a la que pertenece el municipio de Tuxpan, en 2010 generó en total \$29,408 millones de pesos, de los cuales 9,703 millones los generó el municipio bajo estudio, es decir arriba del 1.5\% de ese PIB estatal.

En términos históricos es de utilidad señalar que dicha riqueza municipal, en función de la población económicamente activa ocupada, durante las últimas dos décadas, en orden de prelación ha corrido a cargo del sector terciario, seguido del sector industrial y primario con una participación porcentual de 67.3, 21.01 y 9.59\% respectivamente (SEFIPLAN, 2016), cuyo

\footnotetext{
${ }^{3}$ Aunque es una manera de aproximarse a las afectaciones, no deja de presentar cierto grado de incertidumbre, eso debido a la complejidad de establecer valores más cercanos a la realidad.

${ }^{4}$ De acuerdo con INEGI (2012), el estado de Veracruz produjo en 2010: \$588,166. 5 millones de pesos. 
personal ocupado, para el año de 2015, se estima en 65,000 empleados dedicados a dichas actividades. Sobre el sector terciario y secundario, cabe recordar que en Tuxpan es la actividad maquiladora y el comercio al por menor la que demanda la mayor cantidad de trabajadores, por lo que el PIB municipal depende de alrededor de 57 mil trabajadores, de los cuales alrededor del 10\% labora dentro de las zonas de peligro por inundación.

Tabla 2. Producto Interno Bruto de Veracruz por regiones, 2010.

\begin{tabular}{|l|c|}
\hline Región & \% \\
\hline Huasteca Alta & 5 \\
\hline Huasteca Baja & 5 \\
\hline Las Montañas & 16 \\
\hline Nautla & 4 \\
\hline Olmeca & 17 \\
\hline Papaloapan & 6 \\
\hline Región Capital & 16 \\
\hline $\begin{array}{l}\text { Región de los } \\
\text { Tuxtlas }\end{array}$ & 3 \\
\hline Sotavento & 19 \\
\hline Totonaca & 9 \\
\hline
\end{tabular}

Fuente: Elaboración propia con base en González y Gallegos (2014)

Otro impacto económico a considerar en este primer escenario es la capacidad instalada, que como activo tiene un valor para la administración municipal de Tuxpan. Por ejemplo, la red que integra el sistema carretero del municipio de Tuxpan, constituido principalmente por calles, avenidas y bulevares, según INEGI (2011b), los cuales suman en total 355 kilómetros lineales, de los cuales, 63 de ellos quedarían anegados si el nivel del mar sube de nivel en cinco metros. Ahora bien, si se acepta el costo promedio ( 25 dólares o 500 pesos) que cuesta un metro cuadrado de asfalto (USDA, 2017), así como el ancho promedio de las calles (8 metros) en riesgo de inundación, tenemos que 500 mil $\mathrm{m}^{2}$ resultantes, tienen un valor de $\$ 252$ millones de pesos, si a esto se suma el valor del resto de la infraestructura pública (aceras, drenaje, gasoductos, alumbrado, agua potable, etcétera), la cifra es de aproximadamente 1.2 mil millones de pesos, (equivalente al 15.6\% del PIB municipal de 2010).

Por otra parte, el valor de los predios urbanizados (327 ha), en riesgo de inundarse con una crecida del mar de cinco metros, cotizado en precios del mercado corriente ${ }^{5}$ es de $\$ 13$ mil millones de pesos (más de 650 millones de dólares), lo que representa el $2.1 \%{ }^{6}$ del PIB de todo el estado de Veracruz del año 2010. Mientras que el valor del resto del territorio del municipio de Tuxpan que está en la misma situación $\left(183.3 \mathrm{~km}^{2}\right.$ ), es de $\$ 733$ mil millones de pesos (unos 36 mil millones

5 Tomando como base $\$ 400,000$ pesos por $100 \mathrm{~m}^{2}$, sin contar el valor de las edificaciones.

${ }^{6}$ Considerando el valor de los bienes raíces, tomando como base $\$ 100,000$ pesos por cada $60 \mathrm{~m} 3$ de construcción (INFONAVIT, 2019), es decir, predios no mayores a 10m2 y de una sola planta, habría que agregar otros $284 \mathrm{mil}$ millones de pesos, o sea otro $73.5 \%$ del citado PIB estatal. 
de dólares), lo que representa un PIB más un cuarto del citado PIB estatal. Además, al recuento descrito se deben adicionar las unidades económicas que operan dentro de la zona de riesgo, es decir, 433 comercios (INEGI, 2019) están en peligro de inundación debido a que se ubican por debajo de la cota de los cinco m, así como 305 ha de agricultura de temporal, pues todas están en riesgo de inundación bajo el escenario señalado (SIAP, 2014).

Recapitulando, la anegación por debajo de cinco metros, de la infraestructura de Tuxpan, equivale a un PIB y un tercio del PIB del estado de Veracruz ${ }^{7}$. Ahora bien, además de los 5 mil trabajadores (ya señalados) afectados directamente por el escenario de cambio climático antes propuesto, es pertinente describir los impactos sociales que los efectos de una crecida del nivel de mar de cinco metros dejarían sobre el territorio de Tuxpan.

\section{Impactos sociales ante el escenario de incremento del Mar}

Sin demeritar las cifras económicas, la demanda de vivienda de interés social es la que podría experimentar los mayores cambios si en algún momento el nivel del agua alcanzara la cota de los cinco msnm. Por ejemplo, adicionalmente a las 3,570 solicitudes de vivienda anuales que en promedio requiere el municipio de Tuxpan (INFONAVIT, 2014), se tendrían que sumar otras 4523 correspondientes a todas aquellas viviendas de dicha demarcación que por su ubicación, próxima o sobre de la cota de cinco msnm están en riesgo, es decir, los impactos secundarios del cambio climático incrementarían un $127 \%$ las necesidades de vivienda, tan sólo en la cabecera de Tuxpan, por la reubicación de la población de la zona cero o zona mayor impacto.

Por otra parte, en términos de vulnerabilidad, es preciso señalar que la margen sur de la desembocadura del río Tuxpan prácticamente es la más riesgosa, en particular los lugares rururbanos (antiguos ejidos) ubicadas en la porción oriental de la demarcación municipal (zona baja menor a cinco msnm), dicha zona de inundación, también puede estudiarse desde lo que Tovar y Vázquez (2017) denominan potamoweack (una parasíntesis que emplean dichos autores para darle mayor fluidez epistemológica a la vulnerabilidad vinculada exclusivamente a una inundación). En ese sentido, usando los datos sociodemográficos de INEGI (2011a), a través de un cociente de localización (Lira y Quiroga, 2009) adaptado para fabricar un índice de vulnerabilidad social se pudo conocer de forma relativa el nivel socioeconómico de las principales manzanas urbanas en situación de potamoweack de la ciudad costera de Tuxpan.

Es decir, de las variables, vinculadas con el fenómeno de la vulnerabilidad social, propuestas por (Cutter et al., 2003) se adecuaron para su aplicación conforme a los datos de INEGI. Con esas 18 variables $^{8}$ se logró saber cuáles de ellas superan la media dentro del conjunto de manzanas urbanas que integran la localidad urbana de Tuxpan. Los resultados de las operaciones estadísticas se agruparon aritméticamente en cuartiles para determinar los niveles del índice de vulnerabilidad social.

\footnotetext{
${ }^{7}$ Como un ejemplo análogo, considérese que, en 2005, los daños materiales causados por el huracán Wilma sobre Cancún ascendieron a 1,752 millones de dólares, 0.21\% del PIB nacional de 2010 (Martínez, 2007).

${ }^{8}$ Densidad de población; densidad arquitectónica; hacinamiento; tasa de vejez; tasa de manumisión; tasa PEI; tasa de nuevos vecinos; minorías étnicas; tasa de discapacitados; bajo nivel educativo; tasa de desempleo; porcentaje de población sin derechohabiencia; porcentaje con viviendas con piso de tierra; carencia de electricidad; carencia de drenaje; viviendas con posibilidad de reservas alimenticias; posibilidad de escape por vía automotriz y carencia de internet.
} 
Al respecto, se puede advertir que de las 56 Áreas Geoestadísticas Básicas (AGEB’s) en potamoweack, se resalta que las colonias: Arboledas, La Calzada, El Paraíso, Jardín, Niños Héroes y Emiliano Zapata (oriente de la mancha urbana) presentan el índice de vulnerabilidad social más alto (Figura 3), se podría decir lo mismo de las colonias El Esfuerzo, Los Pinos y La Esperanza (ubicado al centro-norte de la ciudad), pero estas últimas se ubica fuera de la zona de potamoweack; otra ageb, dentro de la zona de estudio, pero con índice de vulnerabilidad social moderado es el que se corresponde con la colonia Revolución.

Es útil señalar que de las 56 AGEB en potamoweack, 4 presentan un índice de vulnerabilidad moderado y 34 más presentan un nivel de vulnerabilidad bajo y otras 15 muy bajo conforme al índice expuesto, lo que las sitúa como las zonas menos vulnerables dentro de ese conjunto (Figura 3). Con los recorridos de campo se puede advertir que las consecuencias del cambio climático se recienten en los sectores urbanos irregulares, principalmente del oriente de la localidad de Tuxpan, donde los problemas sociales continúan creciendo.

Fuente: Elaboración propia con base en (INEGI, 2011b).

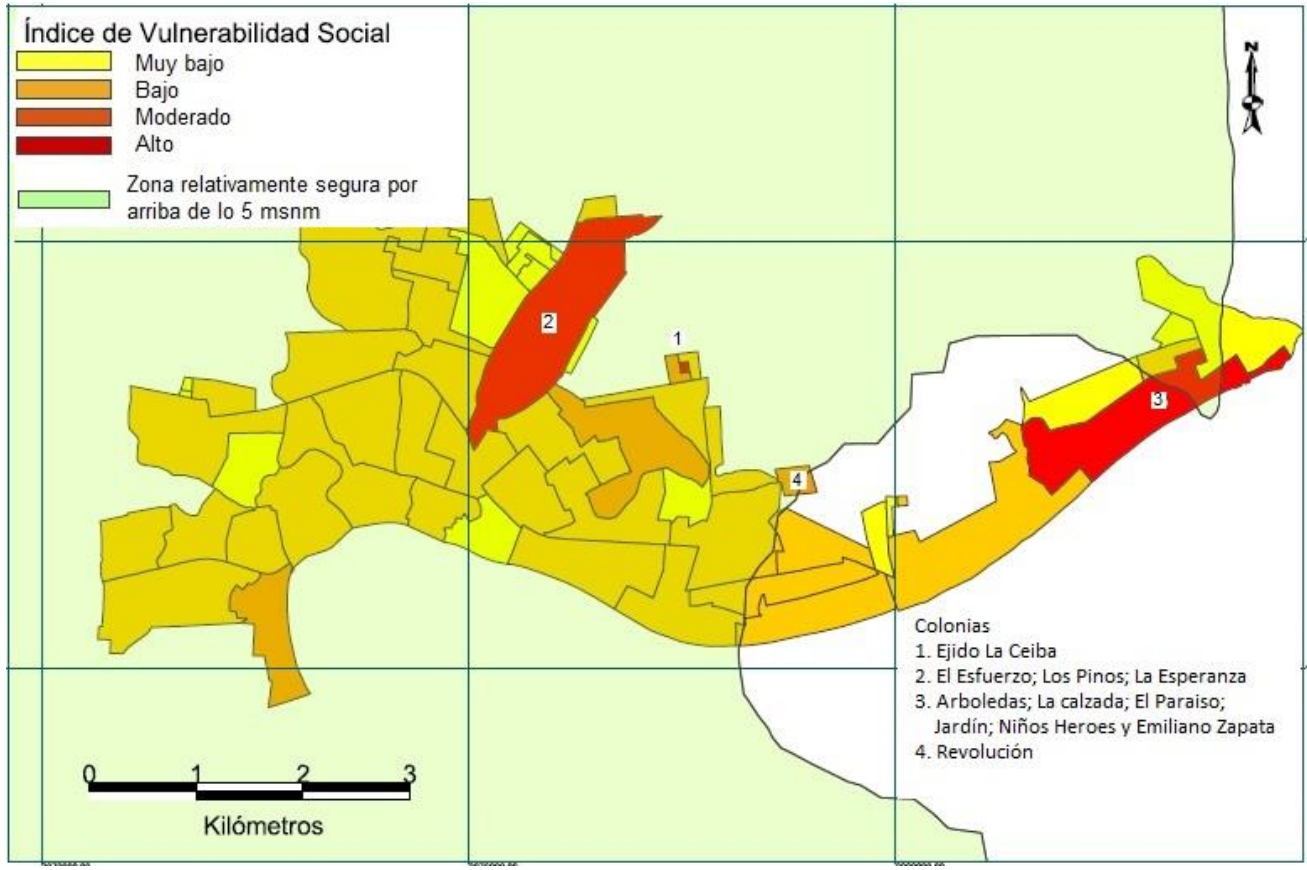

Figura 3. Índice de Vulnerabilidad Social de las zonas por debajo de los cinco msnm de Tuxpan, Veracruz.

El resto de las zonas bajo estudio, en las colonias colindantes al centro geográfico de la ciudad de Tuxpan, inexplicablemente pese a que sus habitantes resienten cada dos años el embate de inundaciones de consideración (como la ocurrida hace dos años, en 2018), la mayoría de ellos, dada su moderada o baja vulnerabilidad social, durante la situación post-desastre, no muestran interés de acudir a un albergue (Figura 4). Además, es preciso destacar que, más del 50\% de los habitantes que viven dentro de la zona de peligro por inundación están imposibilitados económicamente para reubicar su residencia. 
Fuente: Elaboración propia con base en trabajo de archivo y de campo, temporadas 2018-2019.

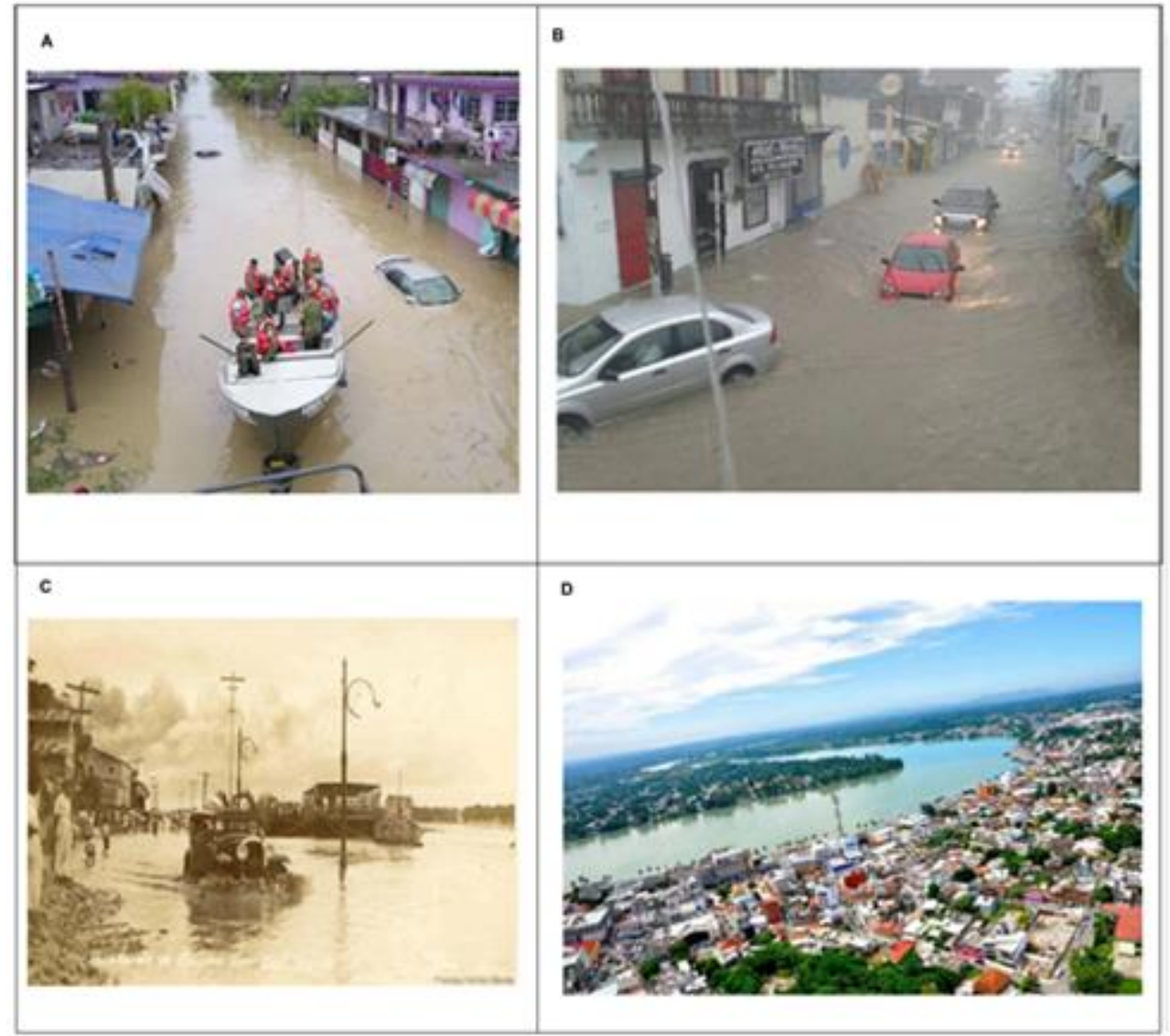

Figura 4. Zonas bajas de la zona cero de Tuxpan: a) plan DN-III en la zona cero de Temapache; b) inundación del 22 de octubre de 2013; c) inundación histórica de 1940; d) panorámica de la desembocadura del río Tuxpan, Veracruz, México.

Se advierte que históricamente las inundaciones de tipo lento, debido al índice de vulnerabilidad social bajo que aglutina más del $87.5 \%$ de las AGEB’s que conforman la zona de mayor peligro por inundación de Tuxpan, no se han percibido en su justa dimensión durante los últimos 50 años, tal cual se ha escenificado en este documento, con inundaciones que alcancen la cota de cinco msnm. Empero, no está de más que la planificación urbana se cimente bajo la consigna de la adaptación a los fenómenos hidrometeorológicos extremos bajo contexto de cambio climático a fin de minimizar calamidades a futuro.

\section{Conclusiones}

Se advierte que el incremento del nivel mar representa un impacto latente a corto plazo, máxime si se considera la combinación de éste con el periodo de retorno de eventos hidrometeorológicos extremos, sobre todo en los puntos más bajos del oriente de Tuxpan, cuyo impacto puede ampliar la damnificación, a la fecha desconocida y minorizada por el gobierno y la población local. No debe banalizarse el latente riesgo por inundación, el cual puede traducirse en el "nuevo Katrina mexicano". Al caso, tomando el escenario de un incremento del nivel del mar de cinco metros en 
el municipio Tuxpan, se estima que un $19.65 \%$ de éste quedaría bajo situación de riesgo por inundación.

Si el aumento de las precipitaciones derivadas por el cambio climático condujeran a un aumento del caudal habitual del río Tuxpan, tal hecho provocaría una crecida de carácter lento en el municipio de Tuxpan, la cual inundaría hasta cinco metros sobre el nivel del mar, donde 187.3 $\mathrm{km}^{2}$ quedarían anegados, de los cuales $0.5 \mathrm{~km}^{2}$, de calles asfaltadas, con un valor de $\$ 252$ millones de pesos se perderían, al igual que otros 1.2 mil millones de pesos de infraestructura pública, sin embargo, la mayor pérdida sería por $\$ 45$ mil millones de pesos correspondientes al territorio urbanizado, sin contar los $\$ 733$ mil millones de pesos que valen sus reservas territoriales. Si se consideran otras entradas como el valor de los bienes muebles de Tuxpan, la catástrofe es enorme, cercana al billón de pesos.

Bajo ese escenario, la merma económica de dicha localidad afectaría al $10 \%$ de las personas ocupadas bajo alguna actividad productiva, pues la mayoría de éstas trabaja cerca o en las zonas que se sitúan por debajo de la cota de los cinco msnm. Como resultado de los impactos sociales, se resalta la necesidad de incrementar la vivienda en más del 127\% para proteger a la población de la zona de análisis. Las colonias tales como Arboledas, La Calzada, El Paraíso, Jardín, Niños Héroes o Emiliano Zapata y El Esfuerzo, Los Pinos y La Esperanza, en el este y centro-norte, respectivamente, presentan un alto índice de vulnerabilidad social, debido a la concentración de población y al material endeble de las viviendas.

Desde otro punto de vista, las principales edificaciones en riesgo hidrometeorológico por cambio climático son: más de 4.5 mil casas, 5 templos religiosos y 50 tiendas de abarrotes. Se estima que alrededor de 18 mil personas viven con una forma de vulnerabilidad moderada en las distintas zonas de peligro por inundación, sobre todo los adultos mayores, puesto que son los que requieren mayor atención durante las inundaciones.

Finalmente, dado que la ciudad de Tuxpan sólo cuenta con el Atlas de Riesgos básicos a escala 1: 250,000, aún hace falta generar más cartografía con el detalle que se presenta, por lo que se considera que esta investigación es un recuento o complemento para la planificación urbana y su respectivo desarrollo local bajo el contexto de cambio climático, con la intención de mejorar la calidad de vida de los habitantes de Tuxpan.

\section{Referencias Bibliográficas}

Alarcón, L. (2011). Análisis de riesgo por inundación en la cuenca baja del río Tuxpan, Veracruz. Tesis de licenciatura, Universidad Veracruzana.

Carreón, D. (1990). Caracterización geotécnica de la planicie costera del Golfo de México entre Tuxpan y Veracruz. Tesis de ingeniería en geología, Universidad Nacional Autónoma de México.

Diario Oficial de la Federación. DOF. (2018). Actualización de la disponibilidad media anual del agua en el acuífero Álamo-Tuxpan (3014), Estado de Veracruz. https://bit.ly/3zai00y

Cruz, C. (2016). Evaluación de la Vulnerabilidad en las costas mexicanas. Tesis de maestría, Universidad Nacional Autónoma de México. 
Cutter S., Boruff B., \& Shirley L. (2003). Social vulnerability to environmental hazards. Social Science Quarterly, 84(2), 242-261. https://doi.org/10.1111/1540-6237.8402002

Ellis, E., Romero, J., Hernández, I., Gallo, C., y Alanís, J. (2012). Evaluación geográfica de áreas susceptibles a inundación en la cuenca del río Tuxpan, Veracruz. Avances en Investigación Agropecuaria, 16(1), 7-28.

Escobar-Ohmtede, A. (2004). Desastres agrícolas en México, catálogo histórico, Tomo II, Siglo XIX (1822-1900). Fondo de Cultura Económica.

Galván, A., y Bertha, A. (2013). Calidad Bacteriológica y Riesgo Sanitario de las playas norte de Tuxpan, Veracruz. Tesis de especialidad, Universidad Veracruzana.

González, A., y Gallegos G. (2014). El producto interno bruto de los municipios de México, II, Estados MZ. Revista Mexicana de Ciencias Agrícolas, 5(8), 1405-1421.

Grinsted, A., Moore, J., \& Jevrejeva, S. (2010). Reconstructing sea level from paleo and projected temperatures 200 to 2100 ad. Climate Dynamics, 34(4), 461-472. doi.org/10.1007/s00382008-0507-2

Instituto Nacional de Estadística y Geografía. INEGI. (2019). Directorio Estadístico Nacional de Unidades Económicas. Aguascalientes. https://bit.ly/3vOE0vM

Instituto Nacional de Estadística y Geografía. INEGI. (2012). Sistema de Cuentas Nacionales de México. Producto Interno Bruto por entidad federativa 2006-2010. Año base 2003. Segunda versión. Aguascalientes. https://bit.ly/3g1ZKO2

Instituto Nacional de Estadística y Geografía. INEGI. (2011a). Resultados definitivos. Censo de población y vivienda 2010. Aguascalientes. https://bit.ly/3wYWPg3

Instituto Nacional de Estadística y Geografía. INEGI. (2011b). Información Vectorial de localidades amanzanadas y números exteriores. Urbanas. Cierre de planeación del Censo de Población y Vivienda 2010. Tuxpan. Aguascalientes. https://bit.ly/3fKfqqa

Instituto Nacional de Estadística y Geografía. INEGI. (2009). Prontuario de información geográfica municipal de los Estados Unidos Mexicanos. Tuxpan, Veracruz de Ignacio de la Llave. Aguascalientes. https://bit.ly/3fPqWRo

Instituto Nacional de Estadística y Geografía. INEGI. (2020). Censo de población y vivienda 2020. Aguascalientes. https://bit.ly/3wVrzhK

Instituto del Fondo Nacional de la Vivienda para los Trabajadores. INFONAVIT. (2019). Casas INFONAVIT y departamentos desde \$319.000. Ciudad de México. https://bit.ly/3gaNqLx

Instituto del Fondo Nacional de la Vivienda para los Trabajadores. INFONAVIT. (2014). Demanda Potencial. (4 bimestre del 2014). Ciudad de México.

Intergubernamental de Expertos sobre el Cambio Climático. IPCC. (2007). Climate Change 2007: Impacts, Adaptation and Vulnerability. Contribution of Working Group II to the Fourth Assessment Report of the Intergovernmental Panel on Climate Change. Cambridge. Cambridge University Press.

Kopp, R., Kemp, A., Bittermann, K., Horton, B., Donnelly, J., Gehrels, R., Hay, C., Mitrovica, J., Morrow, E., \& Rahmstorf, S. (2016). Temperature-driven global sea-level variability in the Common Era. Proceedings of the National Academy of Sciences, 113(11), E1434-E1441. doi.org/10.1073/pnas.1517056113 
LARED. (2019). DesInventar, Sistema de Inventario de Desastres, Versión, 10.02.009. Cali LA RED, corporación OSSO y UNDRR.

Lira, L., y Quiroga, B. (2009). Técnicas de análisis regional. Comisión Económica para América Latina y el Caribe, División de Desarrollo Sostenible y Asentamientos Humanos.

Martínez, M. (noviembre 8, 2007). Los 10 desastres más costosos en México. Expansión. https://bit.ly/3vNOED7

Secretaría de Finanzas y Planeación. SEFIPLAN. (2016). Sistema de información municipal. Cuadernillos municipales 2016. México: Gobierno de Veracruz. https://bit.ly/3g6oyoa

Servicio de Información Agroalimentaria y Pesquera. SIAP. (2014). Datos KMZ abiertos. Cobertura Nacional de frontera agrícola Serie II. México. Frontera Agrícola Región centroeste. https://bit.ly/3yXMbYH

Secretaría de Protección Civil. SPC. (2011). Atlas Municipal de Riesgos nivel básico. Tuxpan. Xalapa. Secretaría de Protección Civil. Gobierno del Estado de Veracruz. https://bit.ly/3uLSOdm

Tovar, R., y Vázquez, S. (2017). Análisis geoestadístico de las muertes por neoplasia en la población de 55 y más años en los municipios del Estado de Veracruz. Universalud, 13(25), 49-58.

United States Department of Agriculture. USDA. (2017). Cost estimating guide for road construction. Washington DC. https://bit.ly/3zaopZE

Vergara, M., Ellis, E., Cruz, J., Alarcón, L., y Galván, U. (2011). La conceptualización de las inundaciones y la percepción del riesgo ambiental. Política y cultura, (36), 45-69.

Veyret, Yvette, Laganier, Richard y BoissieÌre, Aurélie. (2013). Atlas des risques en France: Preìvenir les catastrophes naturelles et technologiques. Paris: Autrement.

Vorderwinkler, B., (1983). La terminal marítima de Petróleos Mexicanos en la Barra Norte de Tuxpan, Veracruz. Tesis de Ingeniería, Universidad Nacional Autónoma de México.

Webster, J., Clague, D., Riker-Coleman, K., Gallup, C., Braga, J., Potts, D., Moore, J., Winterer, E., \& Paull, C. (2004). Drowning of the $-150 \mathrm{~m}$ reef off Hawaii, A casualty of global meltwater pulse 1A? Geology, 32(3), 249-252. doi.org/10.1130/G20170.1

Zavala-Hidalgo, J., Buen-Kalman, R., Romero-Centeno, R., y Hernández-Maguey, F. (2011). Tendencias del nivel del mar en las costas mexicanas. En Alfonso, B., Villanueva, S., Gutiérrez, J., y Rojas J. (eds.), Vulnerabilidad de las zonas costeras mexicanas ante el cambio climático. Campeche, Gobierno del Estado de Tabasco, Semarnat-INE, UNAMICMyL, Universidad Autónoma de Campeche, pp. 315-333. 